\title{
COVID-19: One Patient at a Time
}

\author{
James A. Colbert, $M D$
}

will never forget the first time I cared for a patient who tested positive for COVID-19. It was March 2020, and I

was evaluating a patient in the emergency department (ED). At the time we knew very little about this virus and how it is transmitted. We had all seen the images from Wuhan, China, and had appropriate fear of the lethality of the virus, but there was not yet a clear understanding as to how best to keep health care practitioners safe as they cared for patients with COVID-19.

That evening I received a page that a middle-aged man who had tested positive for COVID-19 was in the ED with fever, cough, and hypoxia. As a hospitalist, my role is to care for these patients, those admitted to stay overnight in the hospital. Before going to see the patient, I watched a video on how to properly don personal protective equipment (PPE). I walked to the ED and suited up with a surgical mask, goggles, disposable gown, and gloves. I was very conscious of the amount of time I spent in that patient's room, and tried to stand at the foot of the bed as much as possible so as to maximize the distance between our faces when we talked.

Upon finishing my assessment, I took off my PPE and exited the room but kept wondering if I had done so correctly. That night when I came home, I slept in the guest bedroom to minimize the risk of transmission of the virus to my wife. For the next 7 days I was terrified that I had been exposed to the virus, worried that I hadn't worn my mask properly, or that I exposed myself to contamination when taking off my goggles and gown. I was hyperaware of my breathing and temperature, wondering if that scratch in my throat was the first sign of something worse. I never did develop any symptoms of illness but the amount of stress I felt that week was enormous.

Over the subsequent weeks I became much more comfortable with putting on and taking off PPE since the volume of COVID patients kept increasing to the point that more than $80 \%$ of the hospital patient census consisted of COVID-19 infections. Those patient interac- tions became less awkward once I could stop worrying about the PPE and focus on providing patient care.

Unfortunately, patient after patient entered the

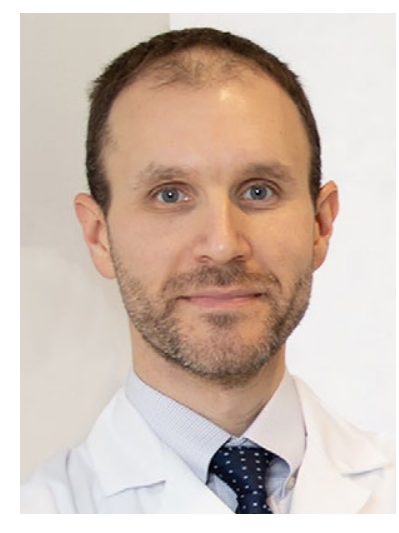
hospital, all with the same symptoms: cough, fever, and hypoxia. Medically there was little decision-making necessary as care was mostly supportive with supplemental oxygen to give these patients time to recover. Instead, I focused on understanding each patient's symptoms and thinking about what could be offered to relieve bothersome symptoms. These patients were isolated in their hospital rooms - denied visitors and their interactions with hospital staff involved layers and layers of protective barrier. I sought to overcome those physical barriers through personal connection - learning about a patient's hobbies, asking about their families, or reminiscing about one of their favorite trips.

Despite this supportive care, many patients ended up intubated in the intensive care unit. Many eventually improved, and we celebrated those individuals - a victory at a time. We even counted the COVID discharges with a running tally; first 10 , then a few dozen, and eventually the number climbed into the triple digits. But not every patient was so fortunate. Hearing about a 40-something who passed away hit too close to home - what if that were me?

The hospitalists I work with rose to the occasion. We feared the virus but still showed up for work because the patients needed us and we had job obligations to honor. Everyone else was stuck at home during lockdown but we still got in our cars and drove to the hospital, suited up in our PPE, and cared for terrified patients that were struggling to breathe.

There was a satisfaction in having a job to do and being able to contribute during this time of global crisis. Staying busy gave our minds something to focus on and helped us feel a sense of purpose. Some of us stayed 
late to coordinate staffing. Others helped to disseminate practice guidelines and clinical knowledge. While others lent a hand wherever they could to pitch in. That sense of camaraderie served as plenty of motivation.

During the early stages of the pandemic, there was a sense that this crisis that would end after a few months and life would return to normal. By May, we experienced a dramatic decline in the number of hospitalized patients with COVID-19, which resulted in a real sense of optimism. But soon it became apparent that this pandemic was not going away anytime soon.

Cases nationwide began rising again over the summer. We saw a steady trickle of new admissions at our hospital month after month until the fall when the rate of admissions accelerated again. The hospital reactivated our surge plan, increased staffing, and confronted the new surge with growing dread. That first surge was all endorphins - but fatigue set in by the time the second wave hit. The volunteerism and sense of "we are in this together" just did not exist anymore. The stories about health care heroes in the broader community waned and the outside world seemingly had moved on from thinking about the pandemic.

Yet we remained, caring for patients with cough, fever, and low oxygen saturation. It was like living through a movie we had already seen before. We knew what we were supposed to do and we followed the script. But now it felt too much like a routine.

It has been a very long 14 months since I first cared for a patient with COVID-19. For much of this time it felt like we were just stuck on a treadmill, passing the time but not making any significant progress towards a postCOVID future state. How many times over this year did we push that date forward in our minds when "life would go back to normal"?

Now, we have reason for hope. More than 100 million Americans have been vaccinated and that number rises daily. The vaccines are remarkably effective, they are making a real difference in reducing the number of patients with COVID-19 at the hospital, and our level of daily anxiety is lower. There is still much uncertainty about the future, but at least we can feel proud of our service over the last year - proud of showing up and donning that PPE. And so, we continue one patient at a time.

Corresponding author: James A. Colbert, MD, Attending Hospitalist, Newton-Wellesley Hospital, 2014 Washington St, Newton, MA, 02462, Senior Medical Director, Blue Cross Blue Shield of Massachusetts; james.colbert@bcbsma.com.

Financial disclosures: None.

doi: 10.1278/jcom.0045

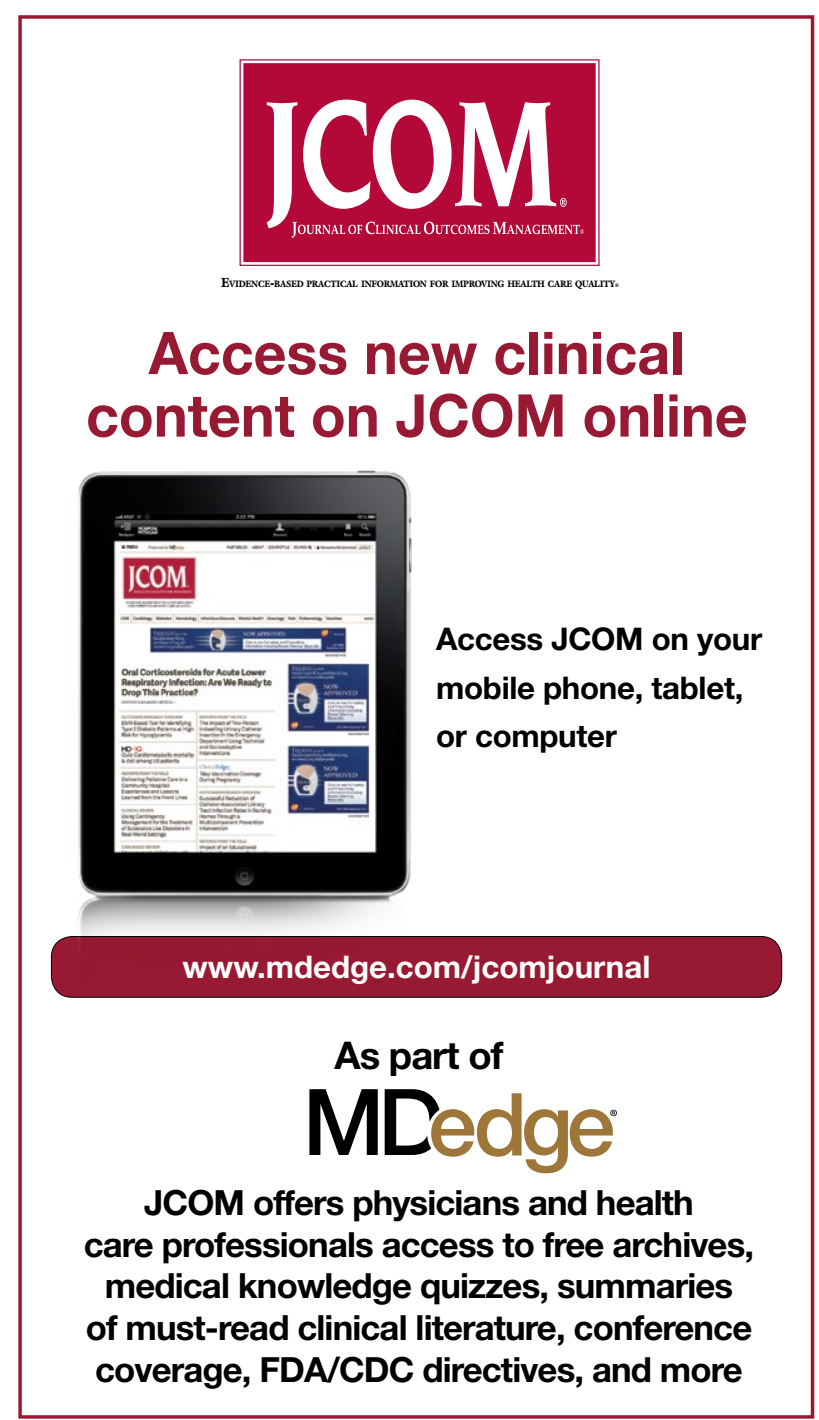

